\title{
Editorial
}

\section{An integrative science approach: neuroscience in the DSM-V and ICD-11}

A key problem in diagnosis is the fact that elaborate classification systems that exist today are solely based on subjective descriptions of symptoms. Such detailed phenomenology includes the description of multiple clinical subtypes; however, there is no biological feature that distinguishes one subtype from another. Moreover, it is recognised that a variety of disorders can exhibit similar clinical symptoms and that one disorder can manifest with distinct patterns in different people (1).

The Diagnostic and Statistical Manual of Mental Disorders (DSM-IV-TR) and the International Classification of Disease (ICD-10), the manuals that specify these diagnoses and the criteria for making them, are currently undergoing revision. These processes are involving a huge numbers of researchers from around the world; it is thus an appropriate time to question if neuroscience is prepared for the DSM-V and the ICD-11, and if they in turn are set for neuroscience. The presence of merely a few number of well-validated biomarkers and the early stage in which our understanding of neurobiology and genetics finds itself have obstructed the integration of neuroscience into psychiatric diagnosis to date. If we integrate a neurobiological approach that describes reliable neurobiological findings based on psychopathological syndrome it will be more solid contrasted to a non-aetiological system of classification. A future diagnostic criteria system in which aetiology and pathophysiology are essential in diagnostic decision making would bring psychiatry closer to other specialties of medicine (2).

The relationship between stress and illness is a strong example of a field of study that can be more fully understood from an integrative perspective. The potential of an integrative approach to contribute to improvements in human health and well-being are more important than historical biases that have been associated with an integrative science approach (3). This approach says very clearly and without a doubt that the causes, development and outcomes of disorders are determined by the relationship of psychological, social and cultural factors with biochemistry and physiology. Biochemistry and physiology are not disconnected and different from the rest of our experiences and life events. This system is based on current studies that reported that the brain and its cognitive processes show a fantastic synchronisation. Consequently, accepting the brain-body-mind complex is possible only when the three systems - nervous, endocrine and immune - have receptors on critical cells that can receive information (through messenger molecules) from each of the other systems (4). The fourth system, the mind (our thoughts, our feelings, our beliefs and our hopes), is part of the functioning of the brain integrating the paradigm. The interaction of the mind, an explicit functioning of the brain, with other body systems is critical for the maintenance of homeostasis and well-being (5).

It is now broadly accepted that psychological stress may change the internal homeostatical state of an individual. During acute stress, adaptive physiological responses occur, which include hyperactivity of the hypothalamic-pituitary-adrenal (HPA) axis. Whenever there is an acute interruption of this balance, illness may result. The social and physical environments have an enormous impact on our physiology and behaviour, and they influence the process of adaptation or 'allostasis'. It is correct to state that at the same time that our experiences change our brain and thoughts, namely, changing our mind, we are changing our neurobiology (6). Of special interest are the psychological stress (stress in the mind) and the interactions of the nervous, endocrine and immune systems. Increased adrenocortical secretion of hormones, primarily cortisol in major depression, is one of the most consistent findings in psychiatry. 
A significant percentage of patients with major depression have been shown to exhibit increased concentrations of cortisol (the endogenous glucocorticoid in humans) in the plasma, urine, saliva and cerebrospinal fluid (CSF); an exaggerated cortisol response to adrenocorticotropic hormone (ACTH); and an enlargement of both the pituitary and adrenal glands $(1,7)$

There is an increasing data supporting that depressive disorders include a group of conditions which may be different with regard to the activity of the HPA axis, immune functions and treatment response. Melancholia, for instance, a syndrome with a long history and distinctive psychopathological features, is differentiated from major depression by the DSM-IV specifiers and partly described in the ICD10th edition. Nevertheless, it has a distinctive psychopathology and biological homogeneity in clinical experience and laboratory test markers, and it is differentially responsive to specific treatment interventions according to international studies (8). In the last few years an important movement proposes to reinstitute the definition of melancholia, set a duration criterion and adds as secondary criteria the associated laboratory findings of dexamethasone nonsuppression of cortisol, high night-time cortisol levels, or decreased REM latency or other characteristic sleep abnormalities (9).

The lack of correlations between clinical and biological data continues to be, according to several authors, one of the great unsolved problems of psychiatry today and could be solved by recovering the value of traditional psychopathological analysis based on fundamental and thorough clinical assessment, which sho uld support aetiological research and treatment decisions.
Mario Francisco Juruena

Department of Neurosciences and Behaviour, Faculty of Medicine Ribeirao Preto, University of Sao

Paulo, Sao Paulo, Brazil

\section{References}

1. Juruena MF, Pariante CM, Papadopoulos AS, Poon L, Lightman S, Cleare AJ. Prednisolone suppression test in depression: prospective study of the role of HPA axis dysfunction in treatment resistance. Br J Psychiatry 2009; 194:342-349.

2. Hyman SE. Can neuroscience be integrated into the DSM-V? Nat Rev Neurosci 2007;8:725-732

3. King SL, Hegadoren KM. An integrative science approach: value added in stress research. Nurs Health Sci 2006;8: 114-119.

4. BASAR E, Karakas S. Neuroscience is awaiting for a breakthrough: an essay bridging the concepts of Descartes, Einstein, Heisenberg, Hebb and Hayek with the explanatory formulations in this special issue. Int J Psychophysiol 2006; 60:194-201.

5. RAY O. The revolutionary health science of psychoendoneuroimmunology: a new paradigm for understanding health and treating illness. Ann N Y Acad Sci 2004;1032:35-51.

6. McEwen BS. From molecules to mind. Stress, individual differences, and the social environment. Ann N Y Acad Sci 2001;935:42-49.

7. Holsboer F, Barden N. Antidepressants and hypothalamicpituitary-adrenocortical regulation. Endocr Rev 1996;17: $187-205$.

8. Juruena MF, Calil HM, Fleck M, Del Porto JA. Melancholia in Latin America studies: a distinct mood disorder for ICD-11. Rev Bras Psiquiatr 2011;33(Suppl. I):S48-S58.

9. TAYLOR MA, Fink M. Melancholia: the diagnosis, pathophysiology and treatment of depressive illness. Cambridge: Cambridge University Press, 2006. 